\title{
EchoGéo
}

46 | 2018

Varia

\section{Géographie et lieux de chant à Shanghai}

\author{
Sarah Defoin-Merlin
}

\section{OpenEdition}

Journals

Édition électronique

URL : https://journals.openedition.org/echogeo/16304

DOI : 10.4000/echogeo.16304

ISSN : 1963-1197

\section{Éditeur}

Pôle de recherche pour l'organisation et la diffusion de l'information géographique (CNRS UMR 8586)

\section{Référence électronique}

Sarah Defoin-Merlin, « Géographie et lieux de chant à Shanghai », EchoGéo [En ligne], 46 | 2018, mis en ligne le 31 décembre 2018, consulté le 01 août 2021. URL : http://journals.openedition.org/echogeo/ 16304 ; DOI : https://doi.org/10.4000/echogeo.16304

Ce document a été généré automatiquement le 1 août 2021

EchoGéo est mis à disposition selon les termes de la licence Creative Commons Attribution - Pas d'Utilisation Commerciale - Pas de Modification 4.0 International (CC BY-NC-ND) 


\title{
Géographie et lieux de chant à Shanghai
}

\author{
Sarah Defoin-Merlin
}

\section{Introduction}

1 À Xi'an, le $1^{\text {er }}$ mai 2017, à l'occasion de la fête du travail, la nouvelle route de la soie et ses retombées économiques espérées ont été célébrées en chanson. La cérémonie, ouverte par le chant "Chanter pour toi » (wei ni fangge), s'est tenue sur le chantier du centre de fret ferroviaire, départ du projet « une ceinture, une route » (yi dai yi lu).

2 Si cet événement éclaire l'importance accordée au chant par le gouvernement chinois dans la promotion de réussites économiques et politiques, il souligne surtout le rapport particulier que chant et espace peuvent entretenir. Le chant, en tant que pratique, est " géographisant » (Raibaud, 2009a, p. 71), c'est-à-dire localisé et localisable. Il peut donc être envisagé comme un médiateur favorisant la compréhension de la société chinoise par son espace. Plusieurs ethnologues, anthropologues et ethnomusicologues ont auparavant envisagé le chant par sa capacité à délimiter et dire un territoire (Roseman, 1998; Koch, 2013), à structurer un groupe (Street, 1993; Reuther, 2007) et à faire émerger une identité collective et individuelle (Garat, 2005). Certains ont aussi considéré plus directement la spatialité du chant en comparant les différents types de chants interprétés en fonction des délimitations d'un territoire donné (Seeger, 2004 ; Olivier, 2004) ou encore en s'intéressant aux lieux spécifiques choisis par les musiciens pour se produire (Picard, 1998). Mais à ce jour, peu d'études se sont interrogées sur ce que la pratique du chant dit de l'espace au sein duquel il est interprété.

Cet article vise donc à souligner l'intérêt d'analyser géographiquement une ville par le chant, notamment dans le contexte chinois, où sa pratique est partagée par le plus grand nombre. En nous basant sur le cas de Shanghai, nous montrerons en quoi les lieux de chant sont des géo-indicateurs de l'organisation spatio-temporelle et socioculturelle de la ville. Les résultats présentés s'appuieront sur une enquête de terrain menée de janvier à mars 2017, au cours de laquelle plusieurs entretiens semi-directifs 
et observations participantes ont été réalisés parallèlement à l'étude de plans d'urbanisme, de cartes touristiques et de sites internet signalant les principaux lieux de chant présents dans la ville. La constitution d'une typologie de ces lieux rendra possibles des analyses pluri-scalaires, qui se baseront à la fois sur l'étude précise du fonctionnement des lieux de chant les plus représentatifs de chaque type et sur leur comparaison. L'objectif principal sera de rendre compte de manière innovante de dynamiques sociales et urbaines déjà connues, tout en en faisant apparaître de nouvelles spécifiquement produites par le chant.

Dans une première partie, nous insisterons sur l'ancrage de la géographie par le chant dans la géographie musicale, puis sur la place centrale que ce dernier occupe en Chine, et particulièrement à Shanghai, avant de présenter et de justifier de manière détaillée notre méthode d'analyse centrée sur les lieux de chant. Nous pourrons ainsi, dans un second temps, mettre en évidence que la répartition des lieux de chant dans la ville, leur implantation précise et leur architecture rendent compte du développement urbain passé et actuel, des politiques culturelles d'internationalisation et de patrimonialisation, ainsi que de la gentrification de l'hyper-centre de Shanghai. Par l'étude de leur temporalité, de leur fréquentation et de leur politique tarifaire nous tirerons des enseignements sur la structuration interne de la société shanghaienne en différents groupes et sur les tensions socio-culturelles qui peuvent exister entre eux. Enfin, nous montrerons que, lorsque l'on parle de ses lieux de chant, Shanghai est perçue par les personnes interrogées comme une ville polycentrale, lieu d'émulation artistique, où les distances sociales et spatiales sont métaphoriquement réduites et dans lequel l'aménagement et la finalité de certains espaces sont modifiés par les activités de chant qui s'y déroulent.

\section{Une géographie de Shanghai par le chant : implications théoriques et méthodologiques}

\section{La géographie par le chant : un ancrage dans la géographie musicale}

5 La géographie musicale, dont la légitimité a longtemps été débattue (Canova, 2013), réussit ces dernières années en France à s'affirmer comme un " champ à part entière " et une « épistémologie féconde dans le tournant culturel de la géographie " (Canova et Raibaud, 2017, p.8). Si des bases de cette géographie ont été établies dès les années 1930, ce n'est que dans les années 1970 qu'un réel mouvement d'étude se structure dans les pays anglophones, puis se diffuse en Europe au début des années 2000 (Guiu, 2007). À ce jour, quatre formes principales de géographie musicale semblent se distinguer tout en se conjuguant (Illustration 1 ). 
Illustration 1 - Les quatre principales approches de la géographie musicale

\begin{tabular}{|c|c|c|c|c|}
\hline Type d'approche & Date d'apparition & $\begin{array}{c}\text { Chercheur ou kcole representatifif du } \\
\text { courant }\end{array}$ & Courant g'ographique infuent & Principe et méthode \\
\hline Géographle de la musique & Années 1970-1980 & \begin{tabular}{|l|} 
Etats-Unis : Ecole de Berkelcy (G.O. \\
Camey) \\
Royaume-Uni : Universités de \\
Nottingham, Bristol ct Durham (D. \\
Matless, R. Hudson, B. Anderson)
\end{tabular} & Cultural Snudies & 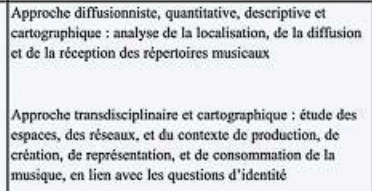 \\
\hline Ǵographie sonore & $\begin{array}{l}\text { Fin des années } 1970- \\
\text { annies } 1980\end{array}$ & D. Pocock, S.J. Smith & $\begin{array}{l}\text { Gíggraphie de la pesception } \\
\text { Geographic humaniste }\end{array}$ & $\begin{array}{l}\text { Etudede de l'immatítialití de la musique : analyse des bruits } \\
\text { et des paysages et espaces sonores }\end{array}$ \\
\hline G'́ggraphic par la musique. & Années 1990-2000 & $\begin{array}{l}\text { J. Connell, C. Gibson, L. Kong } \\
\text { France : J.M. Romagnan, Y. Raibaud }\end{array}$ & \begin{tabular}{|l|} 
Cuftural turn \\
Gender Srudies
\end{tabular} & 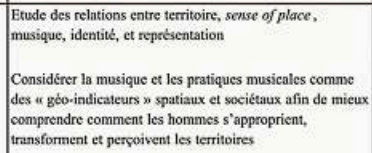 \\
\hline Gémusique & Années 2010 & Y. Raibsuud, N. Cantova & & 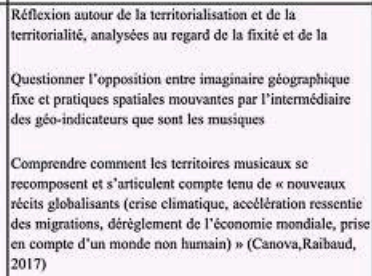 \\
\hline
\end{tabular}

Sources : Guiu, 2006, 2007 ; Raibaud 2006, 2009a et b ; Canova, 2014a et b ; Romagnan, 2003 ; Kearney, 2010. Auteur : S. Defoin-Merlin, avril 2018.

6 La géographie musicale se trouve donc à la croisée de plusieurs géographies : « analyse spatiale, [..] géographie régionale » (Raibaud, 2009a, p. 70), " géographie du pouvoir, sociale, culturelle, du lieu et des représentations, [...] géopolitique [...] emotional geography, géographie du quotidien, théories non représentationnelles, gender studies, postcolonialisme, économie territoriale » (Canova, 2014b, p. 1). La lecture de Shanghai par le chant, ici proposée, développera surtout les volets urbain et socio-culturel d'une géographie par la musique.

\section{Le chant : une activité structurante en Chine}

7 Chaînes d'État consacrées à l'opéra chinois (CCTV 11) et à la musique (CCTV 15); concours de chant télévisés remportant un franc succès populaire (Huang, 2011); chorales de retraités dans les parcs publics (Richaud, 2016; Rochot, 2017) ; karaokés fréquentés régulièrement par tous (Fung, 2009); jeunes écoutant des chanteurs populaires plusieurs heures par semaine (Ho et Law, 2015) ou passants chantant dans la rue : le chant, en Chine, est relié à tous les aspects de la vie (Liang, 1985). Son étude peut donc révéler plusieurs caractéristiques des recompositions socio-spatiales à l'œuvre dans le pays.

8 L'importance attribuée par Confucius à la musique (Yue, 2008) peut encore aujourd'hui expliquer l'utilisation de chansons enfantines dans la transmission des valeurs sociétales chinoises (Chen-Hafteck, 2008). La valorisation de soi associée au chant (Graves, 1946), utilisée aujourd'hui notamment dans l'accession aux études universitaires (Leung et Xie, 2011), justifie aussi l'intérêt porté par la population chinoise au chant. Mais, si le chant a une place si prégnante dans la vie quotidienne des Chinois, c'est sans doute à cause de son utilisation par le gouvernement comme instrument de pouvoir et outil de propagande, en particulier dans les écoles. 
Depuis les années 1980, les jeunes Chinois suivent une à deux heures de cours de musique par semaine avec pour principale activité l'apprentissage du chant (Larson, 1980) et participent, généralement en plus, à un ou plusieurs des ensembles vocaux présents dans les écoles et les universités (Ho et Law, 2011). La quasi-totalité des Chinois est donc en contact avec le chant «à un âge où il est susceptible d'être mémorisé et d'affecter le plus leur vie » (Rogers, 1989, p. 44), d'où leur attachement à cette activité. C'est suite à la fondation de la République de Chine en 1911 et la volonté de Cai Yuanpei (en charge de l'éducation) de «cultiver chez les enfants le sens de la beauté et la bonté de caractère " (Lau, 2005, p. 37) que l'apprentissage de chansons empreintes «de patriotisme, d'autodiscipline, [...] de défense de la nation, et de civisme » (Ho, 2003, p. 290) s'est démocratisé. Depuis 2009, outre « le patriotisme, la cohésion nationale, l'unité et l'harmonie sociales, la mémoire collective et l'héritage culturel » les chansons dans les écoles célèbrent aussi le respect de l'environnement et «l'amour des enfants pour le monde» (Ho et Law, 2011, p. 380), éclairant ainsi les nouveaux enjeux que la Chine se donne.

Le chant sert également au pouvoir en place à modeler les consciences de son peuple et à affirmer sa politique. Dès l'empire des Zhou (-1046 à -221), les chants populaires étaient réarrangés par le Ministère de la musique puis donnés en spectacle pour « rappeler la composition pluriethnique de la Chine » (Trébinjac, 1990, p. 65). Mais c'est certainement la période maoïste (1949-1976), durant laquelle «les chansons pour le peuple» étaient diffusées «dans les espaces publics via des radios et des hautparleurs » (Bryant, 2005 ; Perris, 1983, p. 17) pour atteindre le plus grand nombre, et en particulier les illettrés (Hung, 1996), qui illustre le mieux l'utilisation du chant comme outil de propagande. Aujourd'hui encore, le chant sert à véhiculer, aussi bien en interne qu'à l'international, l'image que la Chine veut se donner. Lors de la commémoration de la fondation de la République populaire de Chine le $1^{\mathrm{er}}$ octobre 2009, étaient ainsi réunis sur la place Tian'anmen des représentants de chacun des 56 groupes ethniques officiels dansant au rythme de leurs chants traditionnels, pour réaffirmer «l'harmonie sociale et le nationalisme" (Ho et Law, 2011, p. 376). Le 8 août 2008, lors de la cérémonie d'ouverture des Jeux Olympiques de Pékin, l'interprétation de l'hymne national chinois et $\mathrm{du}$ chant patriotique "Hymne à la mère patrie"(Ge chang zu guo) par des représentants de chacun des groupes ethniques avaient permis à la Chine d'affirmer l'unité de sa population au niveau international. La chanson "Amis pour toujours" (Yongyuan de pengyou) interprétée par 2008 chanteurs à destination de spectateurs venus de partout dans le monde était aussi un moyen pour la Chine d'envoyer «au monde, un message d'harmonie internationale » (Ho et Law, 2011, p. 384).

\section{Étudier Shanghai par le chant : une méthode centrée sur les lieux de chant}

11 Le choix de Shanghai comme cas d'étude répond à plusieurs critères. Historiquement lieu de plusieurs innovations musicales, c'est à Shanghai que, dans les années 1920, la commercialisation des chansons populaires chinoises débute (Lau, 2005). Le premier conservatoire de musique de Chine y est ensuite construit en 1927 (Bernard, 2007). En 1931, les premières chorales militant pour la libération nationale s'y constituent avant d'essaimer dans d'autres villes (Hung, 1996). Shanghai est également la seule ville dans la Chine occupée où la tenue de concerts se poursuit durant la guerre sino-japonaise 
(1937-1945) (Graves, 1946) et où, en 1998, le premier grand théâtre du pays est ouvert au public (Jiang, 2010). C'est au conservatoire de musique de Shanghai que s'ouvre la première formation du pays incluant l'étude d'instruments de musique moderne et des percussions en 2001; puis, en 2005, ce sont les nouveaux programmes scolaires intégrant des chansons populaires, aussi bien chinoises qu'occidentales, qui y sont appliqués en premier (Ho et Law, 2011). Le gouvernement municipal de Shanghai témoigne également, depuis les années 2000, d'un intérêt particulier pour la culture en termes de planification urbaine. Dès 2004, les autorités municipales ont choisi de réhabiliter certains espaces urbains délaissés, notamment des sites industriels désaffectés, en les reconvertissant en cluster créatifs et culturels (Li et Wang, 2009). Le $13^{\mathrm{e}}$ plan quinquennal de la ville (Shanghaishi shisanwu guihua) (plan 2016-2020) réaffirme cette volonté de s'appuyer sur la culture pour favoriser la régénération urbaine et faire de Shanghai une métropole culturelle internationale ( $\mathrm{He}, 2017)$. La construction de nouvelles infrastructures culturelles est ainsi prévue, en particulier le long du Huangpu, ainsi que la rénovation de celles plus anciennes, notamment de nombreux théâtres, fermés pour travaux, depuis mars 2018. La question de ce que le chant et les lieux qui y sont consacrés disent plus généralement de la structuration et du fonctionnement de la ville et de la société shanghaiennes se pose alors.

Shanghai, comme de nombreuses autres villes chinoises, connait, notamment depuis les années 1990, de très rapides transformations socio-culturelles et spatiales qui vont de pair avec l'avènement d'une consommation de masse et d'une société de loisirs. Conjugués à la décollectivisation urbaine entamée dans les années 1990, ces bouleversements sociétaux font naître un besoin de nouveaux espaces publics voire surtout de nouveaux lieux de sociabilité (Sanjuan, 2003). Dans les années 1980 se multiplient les grands hôtels, puis les parcs publics dans les années 1990 (Rochot, 2017). Depuis les années 2000, de nombreux centres commerciaux se développent, au sein desquels les chaînes de cafés internationales constituent de véritables "lieux de sociabilité [...] et points de rendez-vous pour les rencontres amicales ou professionnelles » (Pons et al., 2006, p. 42), tout comme les restaurants et karaokés qui y sont également implantés (Sanjuan, 2009). Parmi ces lieux de sociabilité, les karaokés des centres commerciaux et les parcs sont des lieux de chant. Le terme « lieu de chant " est ici entendu comme une portion d'espace définie par un groupe de personnes exerçant une activité particulière - ici le chant - et délimitée de manière concrète, que ce soit par les murs d'un bâtiment, des arbres, des poteaux électriques ou encore plus simplement par le cercle des auditeurs. Les lieux où le chant peut trouver un théâtre d'expression spontanée, ainsi que ceux où l'on peut aller écouter en direct les artistes en chair et en os et non par l'intermédiaire de bandes-son, ont ici été privilégiés, en excluant les lieux de culte, les lieux de formation : conservatoires et écoles de musique, plus difficilement accessibles, et les lieux moins conventionnels, comme les stades ou les hôpitaux. Étudier les lieux de chant à Shanghai, c'est donc montrer en quoi ces lieux sont des géo-indicateurs de la ville. Autrement dit, c'est rendre compte de ce que la répartition, l'implantation, l'aménagement, la fréquentation, l'appropriation et la perception des lieux de chant, ainsi que la pratique de la ville engendrée chez les habitants par le chant, disent de l'organisation spatio-temporelle et socio-culturelle de Shanghai.

Dans cette perspective, les lieux de chant les plus usités à Shanghai ont été classifiés en cinq grands types (Illustration 2) : parcs publics (gongyuan), centres culturels (wenhua 
zhongxin), théâtres offrant des représentations d'opéras chinois et/ou occidentaux, karaokés, et bars de styles ancien (reprenant des chansons majoritairement jazz et blues) ou nouveau (proposant des répertoires de chant plus modernes et appréciés surtout par les jeunes générations). Cette typologie a été établie à partir d'un inventaire des lieux de chant présents à Shanghai réalisé à l'appui des plans d'urbanisme et touristiques de la ville, du recensement de ces lieux sur les sites internet officiels des différents arrondissements et sur des sites touristiques en langues chinoise, anglaise et française. Elle a ensuite été complétée et détaillée d'après la mention et la caractérisation des lieux faites par les chanteurs (en grande partie amateurs), les spectateurs, les responsables ainsi que les passants présents aux abords interrogés au cours de 84 entretiens et d'observations participantes menés du 31 janvier au 31 mars 2017 dans la ville-centre-de Shanghai.

Illustration 2 - Une typologie des principaux lieux de chant à Shanghai

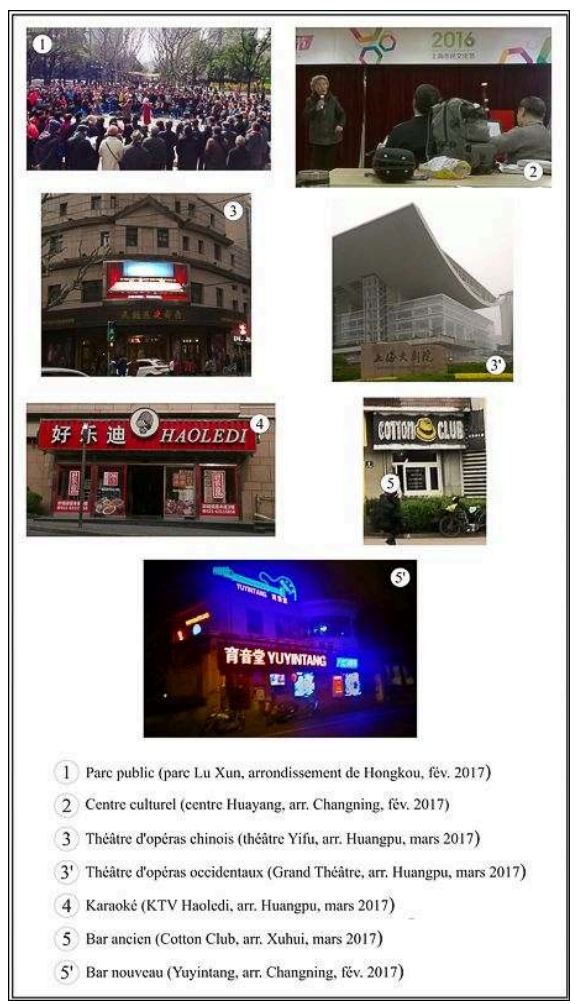

Source : enquêtes de terrain, février-mars 2017. Auteur : S. Defoin-Merlin, avril 2018. 


\section{Appréhender l'organisation spatio-temporelle et socio- culturelle de Shanghai par l'étude de ses lieux de chant}

\section{Une organisation urbaine révélée par les lieux de chant}

À l'échelle de la ville : des lieux témoins de l'histoire et de l'image que Shanghai se donne à l'international

La plupart des lieux de chant de la ville-centre de Shanghai se concentrent à l'Ouest du fleuve Huangpu (Puxi), notamment au niveau des arrondissements de Changning, Xuhui, Jing'an et Huangpu, alors qu'ils sont bien moins nombreux dans sa partie Est (Pudong). Une telle disparité entre les deux rives rend compte d'un aménagement urbain historiquement différencié. La zone où se concentrent les lieux de chant correspond aux anciennes concessions française et internationale (Illustration 3). Durant cette période (1843-1946) de nombreux bars de jazz, sur le modèle des clubs anglais, et de nombreux théâtres y ont été construits à l'initiative des résidents étrangers (Bergère, 2002). Des théâtres d'opéra chinois sont aussi présents en nombre car l'ancienne concession internationale, bien que sous contrôle étranger, abritait $98 \%$ de Chinois (Jiang, 2010). Par comparaison, le peu de lieux de chant à Pudong révèle un développement plus tardif, entamé uniquement dans les années 1990, visant à faire de cet arrondissement, longtemps agricole, puis lieu d'implantation de quelques industries, un centre économique et financier international (Marton et $\mathrm{Wu}, 2006$ ). Les quelques lieux de chant qui s'y trouvent tout de même, en particulier le Centre des arts orientaux de Shanghai (SHOAC) (Shanghai dongfang yishu zhongxin) ouvert fin 2004, sont cependant le signe d'une politique de développement, qui, depuis une dizaine d'années, se veut plus culturelle (Demonet, 2013).

Illustration 3 - La répartition spatiale des principaux lieux de chant dans l'hyper-centre de Shanghai en 2017

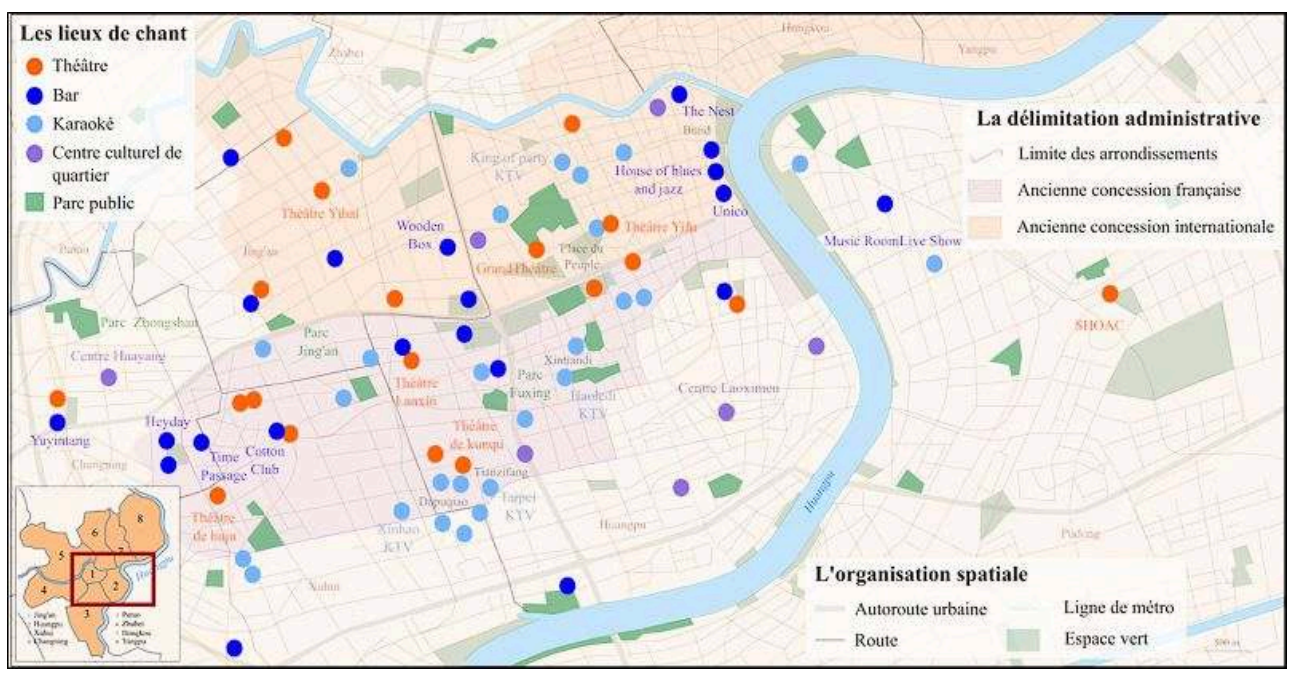

Sources : http://shggwh.com/CentreSearch.html ; http://www.shanghai.gov.cn/; http:// www.smartshanghai.com ; http://www.timeoutshanghai.com/ ; http://www.cultural-china.com/ ; carte du Bureau touristique de la Municipalité ; Sanjuan, 2009. Auteur : S. Defoin-Merlin, avril 2017. 
15 actuelle de Shanghai. La présence, à environ 600 mètres l'un de l'autre, du Grand Théâtre (Shanghai da juyuan), considéré comme « une structure culturelle symbolique » (Kong, 2009, p. 4), et du Théâtre Yifu (Shanghai tianchan yifu wutai), réputé comme hautlieu de l'opéra traditionnel chinois à Shanghai, au niveau de la Place du Peuple, où se trouvent également le gouvernement municipal, le musée d'urbanisme et le musée de Shanghai, marque ainsi le centre choisi de la ville.

Cette proximité du Grand Théâtre, qu'une des responsables du Bureau culturel de l'arrondissement de Huangpu présente comme « lieu de nouveauté et de modernité » et " manifestation d'une culture occidentale », et du Théâtre Yifu, perçu comme « lieu de perpétuation d'une tradition", peut aussi être lue comme signe de la volonté municipale d'exprimer une identité urbaine shanghaienne empreinte d'hybridité entre Chine et Occident, tradition et modernité. Le Grand Théâtre, par son architecture grandiose (Illustration 4), dont la conception en 1994 s'inscrit dans la plus grande autonomie accordée à la ville depuis 1992, est également un symbole du renouveau et de la stratégie d'internationalisation de Shanghai.

\section{Illustration 4 - L'architecture du Grand Théâtre : un reflet de l'identité shanghaienne voulue par la} Municipalité

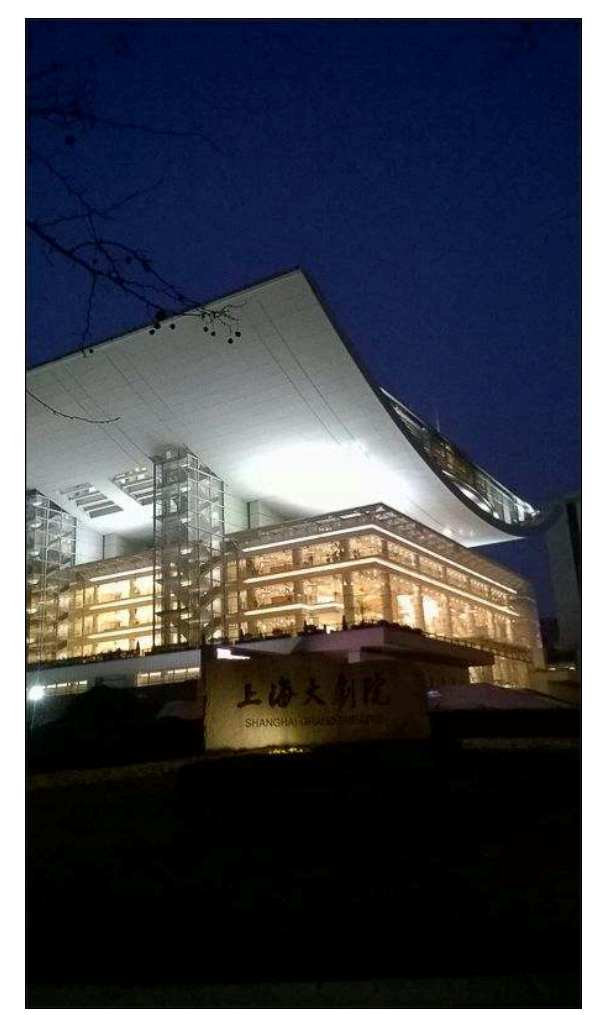

L'architecture du Grand Théâtre a été officiellement pensée pour « reprendre les éléments forts de la culture traditionnelle chinoise interprétés dans un langage contemporain », dont l'expression serait la base carrée et le toit en arc de cercle, symboles traditionnels de la terre et du ciel, associés à une structure en verre synonyme de modernité

Source : Présentation du Grand Théâtre faite par le groupe Arte Charpentier sur leur site internet http://www.arte-charpentier.com/fr/projet/opera-de-shanghai/. Auteur : S. Defoin-Merlin, février 2017.

EchoGéo, 46 | 2018 


\section{À l'échelle d'un quartier : des lieux de chant révélateurs des dynamiques urbaines locales}

17 La présence, l'absence ou encore la fermeture de lieux de chant sont autant de marqueurs des dynamiques urbaines à l'œuvre dans un quartier. Celui formé par les rues Yan'an ouest au Nord, Changshu à l'Est, Fuxing ouest et Huaihai au Sud ainsi que Wukang et Zhenning à l'Ouest a été choisi pour son apparente abondance de lieux variés dédiés au chant (Illustration 5). Cependant, plusieurs se sont révélés fermés : le karaoké de la rue Wulumuqi cédé en 2015, le Cotton Club fermé définitivement le 25 mars 2017 ainsi que le centre culturel d'arrondissement en rénovation.

Illustration 5 - Un quartier où se concentrait initialement une diversité de lieux de chant

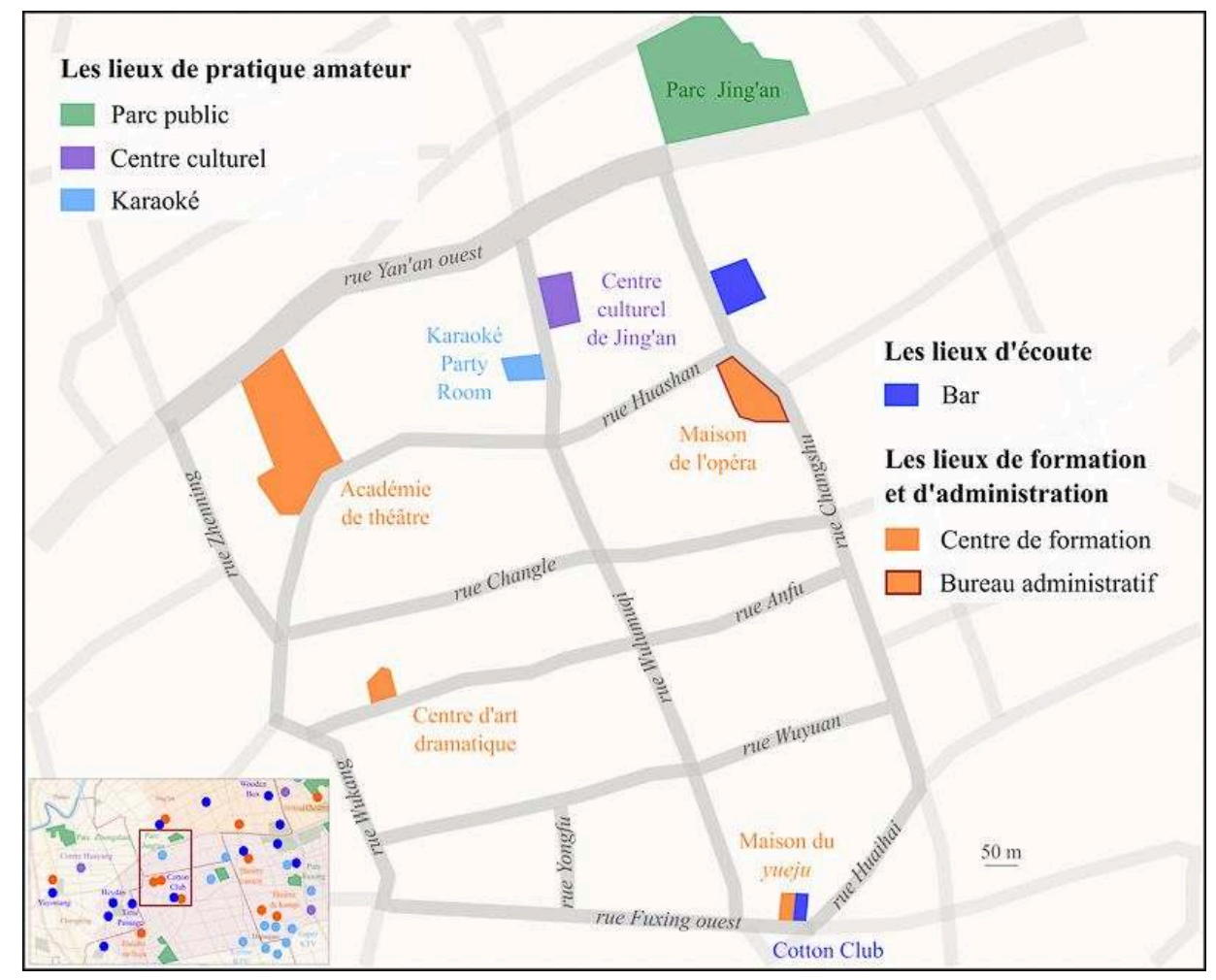

Le quartier formé par les rues Yan'an ouest, Changshu, Huaihai, Fuxing ouest, Wukang et Zhenning Sources : https://map.baidu.com ; enquêtes de terrain, février-mars 2017. Auteur : S. Defoin-Merlin, mai 2018.

Ces fermetures sont le signe du phénomène de gentrification à l'œuvre dans ce quartier de l'hyper-centre, mais aussi de la politique culturelle de la ville. Un couple de chanteurs de jazz et de blues s'inquiète ainsi du fait que « le centre-ville est de plus en plus cher et cela pourrait, à terme, conduire à la disparition de la scène musicale à cause de l'impact que cela a sur les lieux dans lesquels les gens chantent». Les bars, mais aussi les karaokés, puisqu'ils ne sont pas directement gérés par le gouvernement, sont soumis à la hausse des prix du marché immobilier, renforcée par la gentrification, ce qui contraint un grand nombre d'entre eux à cesser toute activité. Les marques dans le paysage urbain de leur fermeture récente s'ajoutent alors aux autres indices visuels d'une gentrification en cours (Illustration 6). 


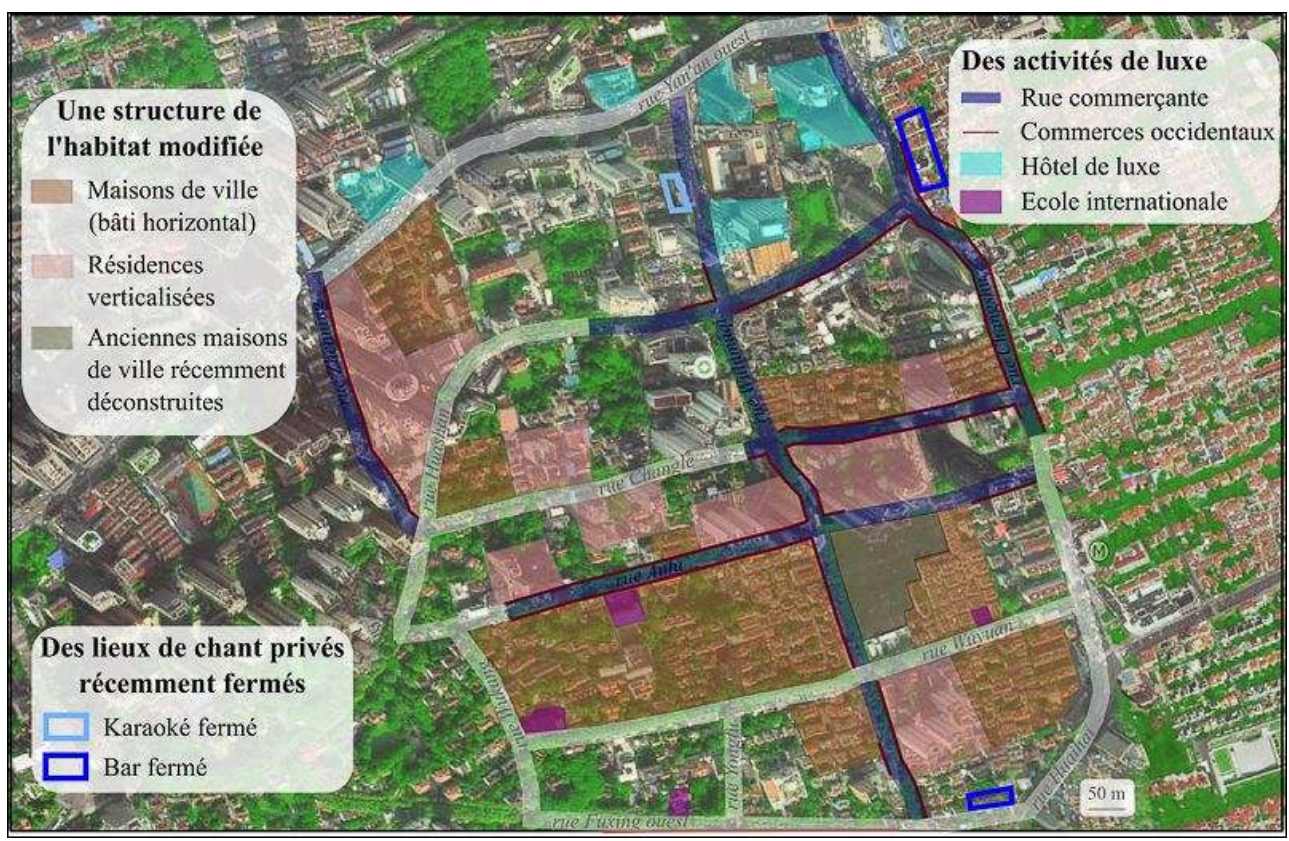

L'étude du quartier formé par les rues Yan'an ouest, Changshu, Huaihai, Fuxing ouest, Wukang et Zhenning, permet de mettre en évidence plusieurs indices, notamment la disparition des bars et karaokés, propres à une gentrification en cours. De nombreux cafés, des magasins de marques occidentales, en particulier de vêtements, des agences immobilières à destination des classes sociales moyennes et élevées, de nombreuses écoles et structures d'accueil internationales pour jeunes enfants aux frais de scolarité élevés, voisinent avec des magasins et restaurants de rue chinois plus modestes notamment dans la rue Wulumuqi, et avec des maisons de ville peu à peu détruites qui laissent place à des résidences verticalisées abordables seulement aux classes les plus aisées.

Sources : https://map.baidu.com ; enquêtes de terrain, février-mars 2017. Auteur : S. Defoin-Merlin, mai 2018.

19 À proximité du quartier étudié, de nombreux autres bars ont aussi été fermés dans la rue Henghsan, le JZ Club dans la rue Fuxing, et le Shelter dans la rue Yongfu. À l'inverse, les bars se multiplient au niveau du Bund ; citons, le bar de jazz Unico ouvert en 2012, le bar-restaurant The Nest qui accueille depuis 2015 des chanteurs rock, ou encore le bar House of Blues and Jazz qui y a été réimplanté en 2008. Selon un journaliste venu récolter les impressions des spectateurs à la veille de la fermeture du Cotton Club, de nombreux bars de type ancien sont réimplantés au niveau du Bund en raison de la "volonté du gouvernement d'y recréer la ville telle qu'elle était dans les années $1930 »$. Cette relocalisation serait alors la traduction d'une stratégie de patrimonialisation visant à faire de cette zone un endroit dans la ville où s'exprimeraient les souvenirs d'une période historique retenue par la Municipalité comme glorieuse et constitutive de l'identité urbaine actuelle (Arkaraprasertkul, 2010). Cette volonté municipale, réaffirmée dans le $13^{\mathrm{e}}$ plan quinquennal, de ressusciter un Shanghai idéalisé des années 1920-1930 n'est pas nouvelle, comme en témoignent la réalisation au niveau de la Place du Peuple de la rue souterraine "Shanghai Street 1930 ", la réhabilitation du quartier Xintiandi envisagé par les autorités municipales comme image du « Paris de l'Orient » (Lau et Wang, 2009) ainsi que la conservation des lilong ${ }^{1}$ de Tianzifang comme signe d'authenticité historique de la ville (Padovani, 2015).

Les Maisons de l'opéra et du yueju ${ }^{2}$ présentes dans le quartier étudié révèlent quant à elles le souci de préservation des différentes formes d'opéras locaux, priorité nationale depuis 2006 (Hewitt, 2011). Cela se traduit à Shanghai par un large soutien financier 
accordé aux Opéras pour l'entretien des établissements et la baisse des tarifs, des campagnes de promotion, et des partenariats entre établissements scolaires et centres consacrés aux opéras provinciaux. La multiplication, depuis 2005, des centres culturels, comme celui de Jing'an, favorise également la pratique de ces opéras par des amateurs, notamment le week-end.

\section{La temporalité des lieux de chant : indice de la structuration interne de la société shanghaienne}

21 La semaine en journée, seuls les parcs, les centres culturels et les karaokés sont animés; en soirée s'ajoutent les bars et les théâtres. L'étude du type de population présente dans ces lieux en fonction des horaires révèle les rythmes de vie variés de différents groupes sociaux. La semaine, en journée, les lieux de chant sont fréquentés uniquement par les retraités, tandis qu'en soirée, ce sont les actifs qui sont présents en nombre. Il faut, cependant, attendre le week-end pour compter de nombreux jeunes (Illustration 7).

Illustration 7 - Les lieux de chant à Shanghai : des lieux fréquentés par des groupes sociaux différents en fonction des horaires

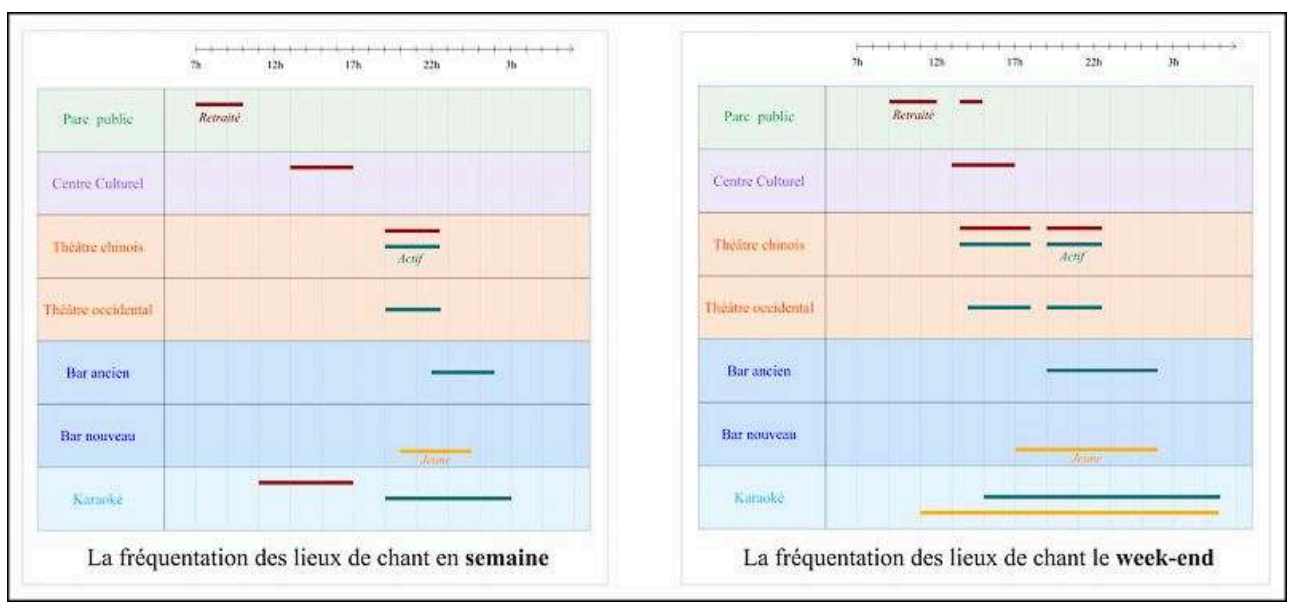

Cette chronologie de la fréquentation des lieux de chant se fonde sur les déclarations des personnes interrogées lors d'entretiens et d'observations participantes, ainsi que sur le planning des activités des lieux étudiés.

Source : enquêtes de terrain, février-mars 2017. Auteur : S. Defoin-Merlin, mai 2018.

Cette temporalité particulière est aussi le signe d'un choix de lieux et d'une perception du chant différents pour chacun des groupes. Les parcs et les centres culturels sont quasi exclusivement fréquentés par les retraités, qui considèrent le chant comme une activité collective salutaire " qui permet de garder la santé ». Souvent véritables lieux de vie - retraités chantent le matin en chorales dans les parcs, se rendent l'après-midi au karaoké ou dans des centres culturels, et participent parfois en plus le soir aux activités annexes organisées par les responsables (dîners, voyages, etc.) - les lieux de chant apparaissent aussi généralement comme remèdes à la solitude. Ce sont, pour certains retraités, les seuls lieux où ils se retrouvent et partagent un thé, une collation, des nouvelles, des photos ou encore le journal.

Les bars anciens et les théâtres, occidentaux ou chinois, sont préférés par les actifs, pour qui le chant est avant tout un passe-temps occasionnel dont ils sont spectateurs. 
Lieux de détente et moyen, selon une cliente du Cotton Club, « de se relaxer après une journée intense de travail et de faire une pause dans la semaine ", ce sont également, des lieux de convivialité où l'« on se retrouve à trois ou quatre pour passer un bon moment entre amis ou entre collègues ", mais aussi des lieux de culture pour ceux qui aiment s'y rendre pour découvrir un style particulier de chant.

Les bars nouveaux accueillent surtout des jeunes de 20-30 ans, pour qui ce sont, en plus des karaokés, des lieux de défoulement où ce qui prime ce n'est pas tant chanter que s'amuser entre amis et "décompresser de la semaine de cours». Cette activité récréative assez occasionnelle est souvent une des rares occasions pour les étudiants, de quitter leur campus universitaire et donc de pratiquer la ville.

\section{Les lieux de chant : une expression des distinctions socio- culturelles endogènes à la société}

Ces façons diverses d'utiliser les lieux de chant soulignent également les distances et les tensions inhérentes à toute société. Si certains d'entre eux sont associés à une génération particulière, c'est généralement parce que les autres ne s'y sentent pas acceptées. Les jeunes et les actifs disent souvent ne pas se rendre dans les parcs pour y chanter car « il n'y a que des personnes âgées ». Tandis que pour les retraités, s'il n'y a pas de jeunes, c'est parce qu' «ils ne partagent pas notre musique, notre culture, et notre passé ». Cette distance intergénérationnelle se retrouve également dans les bars anciens, où seuls les 40-60 ans sont les plus présents. Mais, c'est certainement dans les karaokés que ce désir d'entre-soi intragénérationnel et d'évitement intergénérationnel s'exprime le mieux. Beaucoup de retraités, alors qu'ils fréquentent régulièrement ces lieux en semaine, indiquent clairement ne pas s'y rendre le week-end du fait de l'affluence des jeunes et des actifs à ce moment-là.

La diversité des genres musicaux et des tarifs que proposent les lieux de chant illustre les différences de revenus et de niveaux de vie de la population chinoise. Si les parcs et centres culturels accessibles gratuitement regroupent des retraités de toutes classes sociales, les gammes de prix progressifs dans les karaokés, les bars et les théâtres, sont l'indice d'une fréquentation diversifiée. À ce titre, le Théâtre Yifu, pour 8 des 19 représentations programmées au mois de mars 2017, proposait des prix d'entrée compris entre 40 et 80 yuans, alors que les tarifs des autres représentations allaient de 80 à 580 yuans. Le responsable du bar Yuyintang indique également veiller à proposer des concerts à prix variés, parfois gratuits, pour que le facteur financier ne constitue pas une barrière.

Mais les lieux de chant, et notamment les lieux d'écoute, permettent aussi à certains de se distinguer socialement. Cette stratégie de valorisation et de distinction commence souvent par l'affichage d'une prédilection, réelle ou feinte, pour un répertoire de chant particulier. En Chine, assister à une représentation d'opéra occidental, c'est s'affirmer comme appartenant aux classes sociales élevées ou aux classes moyennes aspirant à le devenir (Demonet, 2013). Le public n'est pas le même non plus en fonction des types d'opéras chinois. Les artistes et responsables des troupes d'opéra interrogés s'accordent à dire que si le jingju est apprécié par l'ensemble des classes sociales, le huju et le yueju s'adressent prioritairement aux classes populaires, tandis que le kunqu, est plutôt «à la portée de gens éduqués qui aiment dire qu'ils ont assisté à une telle représentation ». Ce choix d'un répertoire particulier entraîne la fréquentation de lieux 
dédiés. Le Grand Théâtre est ainsi perçu par de nombreux Shanghaiens comme un lieu réservé aux classes sociales les plus aisées (Kong, 2009). Le choix de la place a aussi toute son importance. Au Grand Théâtre et au Théâtre Yifu, comme dans la majorité des théâtres, les meilleures places sont les plus chères (Illustration 8). Être à une bonne place, c'est donc aussi afficher son appartenance sociale. La salle de spectacle devient

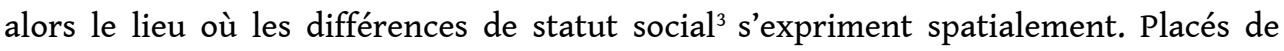
manière à mieux voir la scène, les spectateurs les plus aisés sont aussi les mieux exposés à la vue des autres. Une distinction vestimentaire, par le port de smokings pour les hommes et de robes de soirée pour les femmes est souvent, de ce fait, aussi opérante.

\section{Illustration 8 - Le théâtre : expression spatiale d'une différenciation sociale}

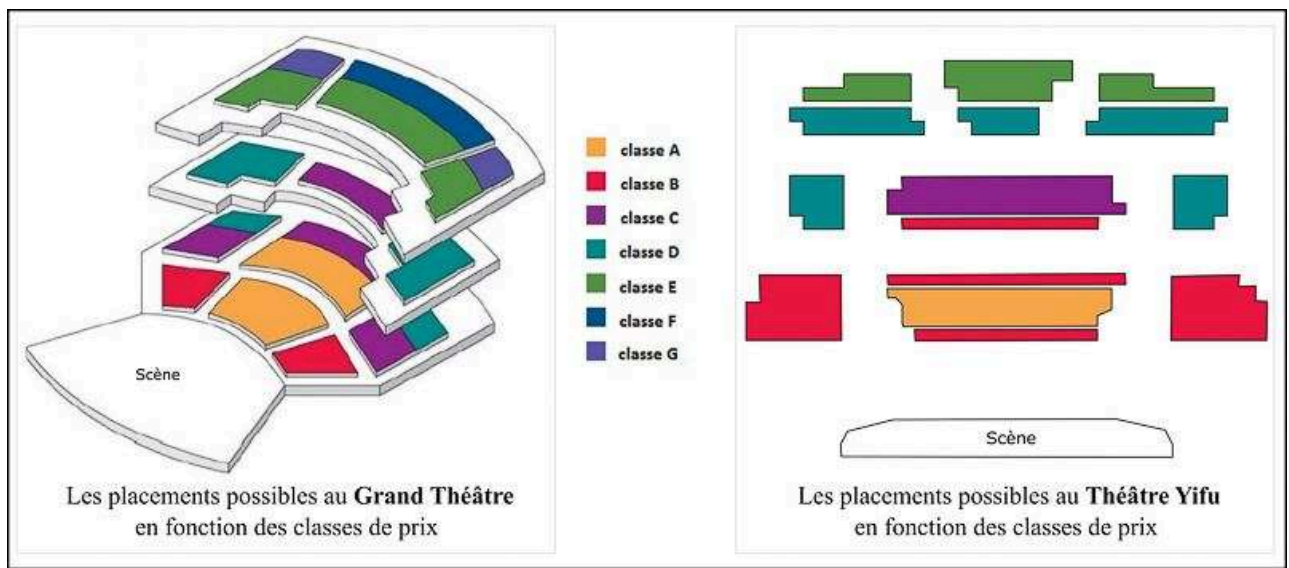

Sources : www.shgtheatre.com (figure gauche); www.tianchan.com (figure droite). Auteur : S. DefoinMerlin, mai 2018

Le caractère parfois illusoire du multiculturalisme shanghaien est également mis en avant par les lieux de chant. Parmi les types les plus répandus à Shanghai, beaucoup ne sont pas fréquentés par les Occidentaux. Si aucun de ceux qui ont été interrogés ne chante dans les centres culturels de quartier, certains écoutent parfois les chorales dans les parcs et seulement quelques-uns se rendent occasionnellement dans des théâtres chinois. Lors de la représentation d'une pièce de jingju au Théâtre Yifu le 5 mars 2017, seuls 11 Occidentaux, parmi les 928 personnes présentes, ont ainsi été dénombrés. Le Grand Théâtre et les bars réunissent plus souvent Chinois et Occidentaux, mais peu d'interactions ont lieu. Cette absence d'interactions réelles s'explique, selon une professeure de musicologie et de chant à l'Université Normale de Shanghai, par la barrière de la langue, le fort turn-over des expatriés, ainsi que la complexité des chants chinois. Les difficultés de l'acculturation et la prégnance de la culture d'origine justifient aussi certainement cette propension de chacun des groupes de nationalité à rester entre-soi.

\section{Le chant : moteur de transformations de la ville shanghaienne}

Puisque les lieux de chant miment la société à plus grande échelle, ils témoignent en partie de l'organisation spatio-temporelle et socio-culturelle de la ville. Mais le chant, 
en tant qu'il modifie la pratique et la perception de la ville que chanteurs et spectateurs ont, donne également à voir une autre image de Shanghai.

\section{Shanghai vue par ses chanteurs : une ville polycentrale ${ }^{4}$, haut lieu d'émulation artistique}

Lorsqu'elle est appréhendée uniquement par le chant, Shanghai revêt une configuration particulière et génère une nouvelle perception de son espace. Quand on interroge les chanteurs et les spectateurs sur la localisation des lieux de chant dans la ville, les théâtres sont associés à la Place du Peuple, les bars de type ancien au Bund et les karaokés à Dapuqiao (Illustration 3). Ces différents secteurs sont désignés comme autant de "centres dans la ville » et sont, par là même, le signe d'une perception et d'une pratique spécifiques de la ville suscitées par le chant, puisque la Place du Peuple n'est plus le seul centre désigné. La répartition des lieux de chant établit alors dans la ville une polycentralité et un découpage sectoriel par type de lieux.

31 Lieu unique de mélange des cultures musicales, où, selon un couple de chanteurs américano-shanghaien, « de l'opéra traditionnel chinois, de l'opéra occidental, du jazz, du rock, du swing, de la country, de la pop, du R\&B, de l'électro, de l'underground, etc » aux colorations variées venant « de partout dans le monde » s'entrecroisent, Shanghai devient elle-même un vaste et diversifié lieu de chant reconnu à l'échelle internationale. De nombreux chanteurs professionnels, originaires de pays variés, voient en cette ville " un endroit unique où il faut être ", " une terre d'opportunité et de créativité où il est plus facile de vivre de son art » et, où « un véritable réseau d'entraide entre musiciens existe» rendant alors possible «un enrichissement permanent du niveau musical ».

\section{Le chant facteur de rapprochement spatial et social}

Le chant a le pouvoir de rassembler en un même lieu des personnes d'horizons variés. À Shanghai, dans la plupart des lieux de chant, se retrouvent des habitants des différents arrondissements. Certains passionnés n'hésitent pas à faire plusieurs heures de trajet pour rejoindre un bar, un parc ou un centre culturel de prédilection. La notoriété du parc Lu Xun et de ses chorales, dans l'arrondissement de Hongkou, attire ainsi des chanteurs de Pudong et Jinshan pourtant situés à plusieurs kilomètres. Mais ce sont certainement les centres culturels, obligés de se spécialiser dans seulement quatre ou cinq des 317 types d'opéra recensés en Chine (Wong, 2007), qui illustrent le mieux cette réduction symbolique des distances entre arrondissements. Certains centres, seuls de la ville à proposer tel ou tel type d'opéra, attirent des amateurs de tous les arrondissements, quel que soit leur éloignement. À titre d'exemple, le centre Chengqiao, dans l'arrondissement de Minhang, seul à proposer des activités de huangmeixi, compte parmi ses chanteurs, des habitants notamment de Pudong, Baoshan, Qingpu et Songjiang. La coprésence symbolique en un même lieu de différents arrondissements s'accompagne dans les théâtres d'une coprésence de provinces et de nationalités diverses. Lors d'une représentation de la Traviata en mars 2017 au Grand Théâtre, se trouvaient ainsi côte à côte, entre autres, des Shanghaiens, des spectateurs des provinces de Shandong, Jiangsu et Anhui, ainsi que des Américains et des Japonais. 
Céduction des distances s'exprime également concrètement par l'aménagement spatial de certains centres culturels. Dans celui de Chengqiao, où le dimanche plusieurs activités d'opéras sont proposées aux mêmes horaires, des cloisons amovibles permettent de délimiter des espaces propres pour chacun des opéras provinciaux pratiqués. Le spectateur est donc invité à passer d'une salle de yuju, à une de wuju puis de huiju et enfin à une de huangmeixi, traversant symboliquement les provinces du Henan, du Zhejiang, de l'Anhui, et du Hubei (Illustration 9).

Illustration 9 - Le centre culturel de quartier de Chengqiao : une réduction spatiale symbolique des distances entre provinces

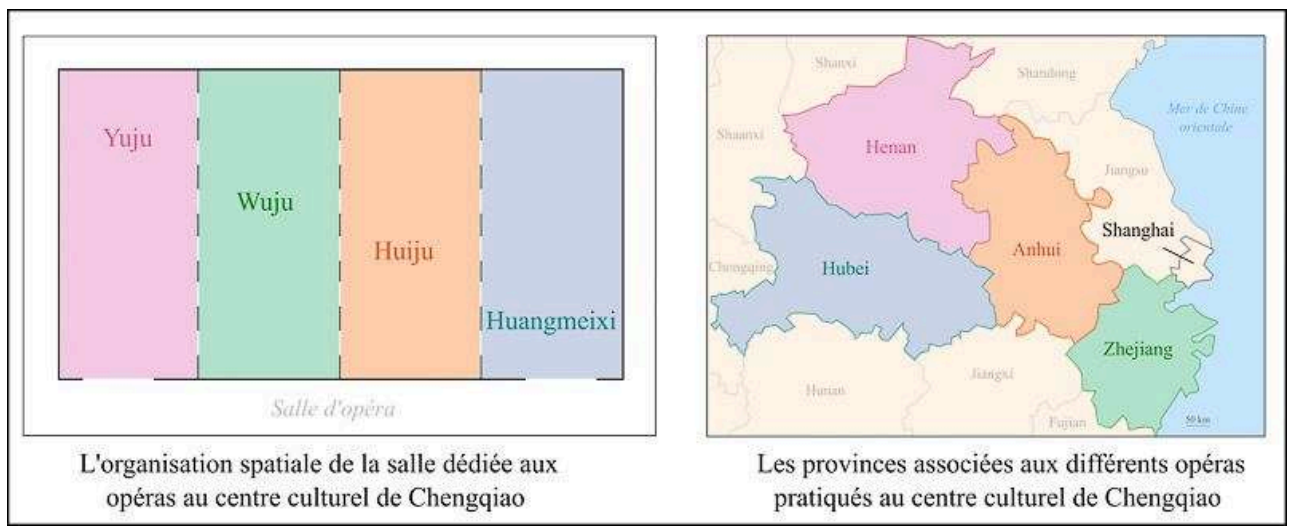

Sources : enquêtes de terrain, février-mars 2017 (figure gauche) ; Sanjuan, 2015 (carte droite). Auteur : S. Defoin-Merlin, mai 2018.

Bien que le chant souligne souvent les différences sociales, il peut aussi les estomper dans certains lieux de pratique. Dans les centres culturels se produit parfois une véritable coopération intergénérationnelle. L'activité huangmeixi du centre de Chengqiao regroupait ainsi le 13 mars 2017, huit chanteuses âgées de 20-35 ans, deux retraitées, trois jeunes de 20-30 ans, deux chanteurs de 30-50 ans et cinq âgés de 50-70 ans. Selon le responsable de cette activité, c'est "grâce au huangmeixi, qu'il y a une rencontre entre jeunes et retraités » car chacun y apporte son expérience; une réelle complicité, pour ne pas dire amitié, s'instaure alors entre participants.

De réels échanges entre personnes de nationalité et de statut différents s'opèrent parfois aussi. La Shanghai International Chorale League (SICL), créée en 2011, se veut figure d'exemple puisque, selon ses fondateurs, elle compte en ses rangs «des chanteurs [amateurs] originaires de 20 ou 30 pays différents qui vivent maintenant à Shanghai. Ils sont dentistes, avocats, cuisiniers, vendeurs, professeurs, etc. C'est donc une grande chorale mixte, qui a la particularité d'être composée à $70 \%$ de chanteurs chinois, alors que dans ce type de chœur il y a le plus souvent une très grande majorité d'Occidentaux ». En chantant ensemble, des personnes qui ne se seraient probablement jamais rencontrées dans d'autres circonstances, sont donc amenées à échanger et coopérer. Ce brassage socio-culturel existe aussi à un niveau plus institutionnel. Un partenariat entre le Conservatoire de musique de Shanghai et le Lycée Français a, ainsi, débuté en 2015 : des étudiants ou professionnels du conservatoire enseignent, entre autres, des techniques de chant aux collégiens et lycéens français, et peuvent en retour juger de la particularité de la mise en scène des opéras comiques français joués par les élèves, de façon à pouvoir les adapter par la suite à un public chinois. 


\section{Une spatialité modifiée par le chant} lesquels elles se déroulent. Le parc Lu Xun est certainement un des lieux de chant à Shanghai où cette transformation s'illustre le mieux. Ce parc répertorié comme gongyuan (littéralement : jardin public) a été pensé par la Municipalité comme espace public aussi bien en termes de statut que d'usage. Son accès est gratuit depuis 2011 et donc en principe ouvert à tous. Ses horaires d'ouverture et de fermeture ont aussi été à la même époque, selon une des responsables du Bureau culturel de l'arrondissement, " adaptés aux besoins de la population ", puisque le parc est maintenant ouvert de $6 \mathrm{~h}$ à $19 \mathrm{~h}$ du 1 octobre au 31 mars et de $5 \mathrm{~h}$ à $19 \mathrm{~h}$ le reste du temps, et non plus jusqu'à $18 \mathrm{~h}$.

Cependant la présence, le dimanche, de nombreuses activités, notamment de chant, aux espaces éphémères, spontanément délimités par les participants et repris de semaine en semaine, fait de ce parc, en pratique, non plus un espace public, mais un espace composé d'une multitude de lieux de sociabilité (Illustration 10).

Illustration 10 - Le parc Lu Xun : un espace public, mosaïque de lieux de sociabilité le dimanche

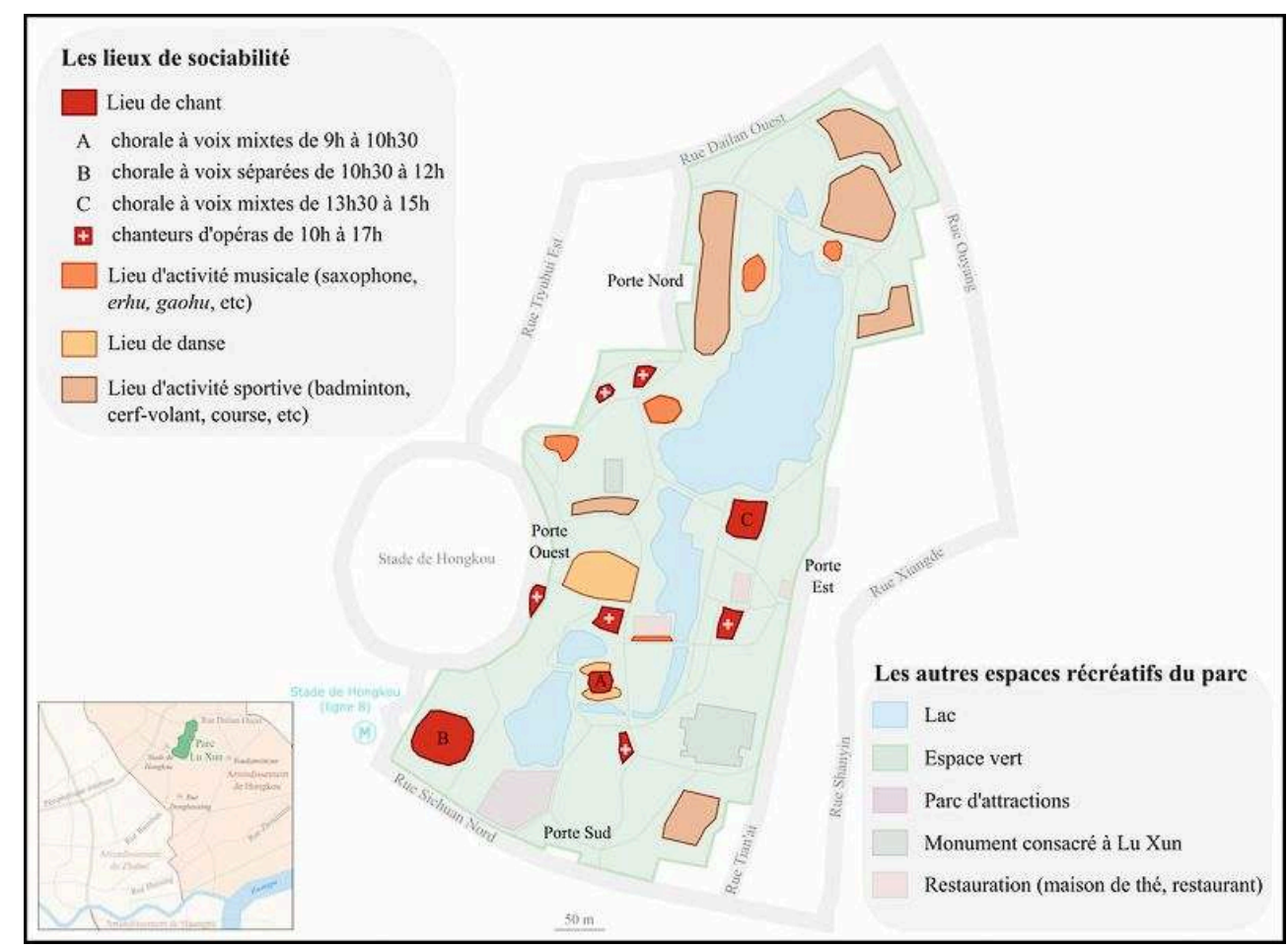

Sources : plan à l'entrée du parc ; enquêtes de terrain, février-mars 2017. Auteur : S. Defoin-Merlin, mai 2017.

Chacun des groupes s'approprie à sa manière une partie de l'espace et en fait de véritables lieux. Le mobilier urbain est détourné de ses fonctions originelles et prend une signification particulière le temps du chant. La première chorale à se produire le dimanche dans le parc fait du pavillon commémoratif consacré à Lu Xun, une scène ; des murets en pierre servant habituellement de bancs, une limite entre chanteurs et spectateurs; des arbres, un pupitre et un porte-manteau; et de la poubelle, une pince de fixation (Illustration 11). Plusieurs portions d'espace du parc sont donc appropriées et aménagées par les participants pour et par le chant. En devenant ainsi lieux de 
chant, ces espaces sont suffisamment normés pour que les chanteurs discutent et partagent ensemble, entre deux chants, aussi bien des expériences, que des photos, des aliments et des cadeaux. L'anonymat caractéristique des espaces publics est dès lors levé, et le parc Lu Xun devient momentanément, mosaïque de lieux de sociabilité.

Illustration 11 : L'aménagement en lieu de chant du pavillon commémoratif consacré à Lu Xun

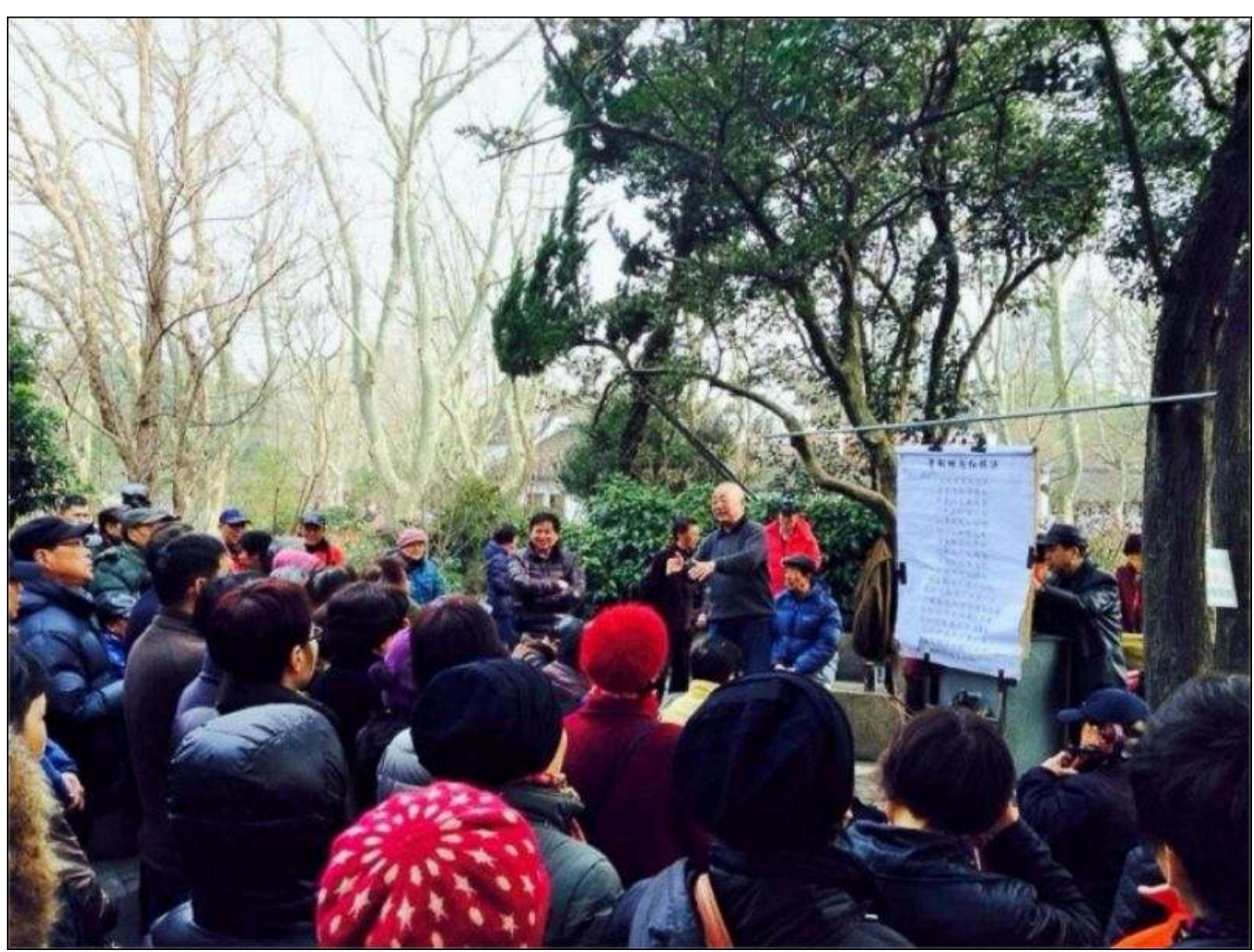

Auteur : S. Defoin-Merlin, février 2017.

\section{Conclusion}

39 En Chine, chanter est une activité courante, appréciée par le plus grand nombre et utilisée depuis plusieurs siècles par le pouvoir en place comme moyen d'expression et outil de propagande. Par sa place centrale et structurante, le chant est pratiqué à divers moments et endroits. Son étude peut donc permettre de comprendre en partie la société dans son espace. La méthode proposée dans cet article pour analyser une ville chinoise par le chant, s'inspire de la géographie par la musique, et se centre sur les lieux d'écoute et de pratique, ici étudiés à Shanghai.

La répartition des lieux de chant dans la ville, leur présence, leur absence ou leur fermeture dans un quartier, ainsi que l'implantation et l'architecture de ceux que la Municipalité considère comme primordiaux, illustrent les politiques culturelles et d'aménagement urbain. Leur temporalité, leur fréquentation et leur fonctionnement sont autant d'indices de la structuration sociale et des distances ou tensions de la société. Puisque le chant et ses lieux sont envisagés par la population comme marqueurs identitaires générationnels, sociaux et nationaux, le rythme de vie, la sociabilité, et les préférences culturelles de chacun de ces groupes sont aussi appréhendés. 
41 En plus de leur capacité à rendre compte de l'organisation spatio-temporelle et socioculturelle formelle, les lieux de chant sont aussi à l'origine d'une appréhension moins systématique de la ville. Shanghai, lorsqu'elle est étudiée uniquement par ses lieux de chant, devient une ville polycentrale sectorisée par type de lieux de chant, et acquiert sur la scène internationale l'image nouvelle de haut lieu d'émulation vocale. La finalité et l'aménagement de plusieurs de ses espaces publics sont également modifiés par la formation puis l'usage de certains lieux de chant. Au sein d'un lieu, l'aménagement spatial et la réunion de personnes d'horizons divers, réduisent symboliquement la distance entre ses arrondissements et entre ses groupes sociaux, ainsi que son éloignement des autres provinces chinoises et des autres pays.

\section{BIBLIOGRAPHIE}

Arkaraprasertkul N., 2010. Power, Politics, and the Making of Shanghai. Journal of Planning History, vol. 9, n 4, p. 232-259.

Bergère M.-C., 2002. Histoire de Shanghai. Paris, Fayard, 520 p.

Bernard M.-H., 2007. Les compositeurs chinois à l'heure de la mondialisation. Études chinoises, vol. 26, p. 235-244.

Bryant L.-O., 2005. Music, memory, and nostalgia: collective memories of cultural revolution songs in contemporary China. China Review, vol. 5, nº 2, p. 151-175.

Canova N., 2013. Music in French geography as space marker and placemaker. Social \& Cultural Geography, vol. 14, n 8, p. 861-867.

Canova N., 2014a. La musique au cour de l'analyse géographique. Paris, L'Harmattan, 213 p.

Canova N., 2014b. Limites et contours de la géographicité des objets « sociaux ». Comment les géographes peuvent-ils s'accorder ?. $20^{\text {ème }}$ Biennale de géographie d'Avignon: Controverses et géographies, Géopoint 2014, 12-13 juin, 2 p.

Canova N., Raibaud Y., 2017. Introduction. Les figures d'attachement dans la géomusique. L'Information géographique, vol. 81, $\mathrm{n}^{\circ} 1$, p. 8-19.

Chen-Hafteck L., Xu Z., 2008. Pulling the River: The Interactions of Local and Global Influences in Chinese Early Childhood Music Education. Arts Education Policy Review, vol. 109, n 3, p. 9-16.

Demonet G., 2013. Les relations musicales entre la Chine et l'Europe depuis les années 1980 : un équilibre délicat entre marché et dirigisme. Relations internationales, vol. 156, n 4, p. 125-137.

Fung A., 2009. Consuming Karaoke in China: Modernities and Cultural Contradiction. Chinese Sociology \& Anthropology, ${ }^{\circ} 42$, p. 39-55.

Garat I., 2005. La fête et le festival, éléments de promotion des espaces et représentation d'une société idéale. Annales de géographie, vol. 643, n 3, p. 265-284.

Graves S.-M., 1946. Music Education in China. Music Educators Journal, vol. 33, n 1, p. 26-27/59-61. 
Guiu C., 2006. Géographie et musiques : état des lieux. Une proposition de synthèse. Géographie et cultures, $n^{\circ} 59$, p. 7-29.

Guiu C., 2007. Espaces sonores, lieux et territoires musicaux : les géographes à l'écoute. Les Cafés géographiques Vox Geographica, 7 p.

He S., 2017. The creative spatio-temporal fix: Creative and cultural industries development in Shanghai, China. Geoforum, $10 \mathrm{p}$.

Hewitt D., 2011. Chinese Society, in European Union National Institutes for Culture - Orientation for Cultural Cooperation Between China and Europe, Europe-China Cultural Compass. Chine, Goethe Institut, p. 120-123.

Ho W.-C., 2003. Westernization and social transformation in Chinese music education, 18951949.History of Education, vol. 32, n 3, p. 289-301.

Ho W.-C., Law W-W., 2011. Music education in China: In search of social harmony and Chinese nationalism. British Journal of Music Education, vol.28, n³, p.371-388.

Ho W.-C., Law W-W., 2015. Popular music and school music education: Chinese students' preferences and dilemmas in Shanghai, China. International Journal of Music Education, vol. 33, $\mathrm{n}^{\circ} 3$, p. 304-324.

Huang Y., 2011. La dynamique culturelle en Chine contemporaine : analyse de phénomène de Super Girl Voice concours de chant. Thèse de l'université de Lyon Jean Moulin, 244 p.

Hung C.-T., 1996. The Politics of Songs: Myths and Symbols in the Chinese Communist War Music, 1937-1949. Modern Asian Studies, vol. 30, n 4, p. 901-929.

Jiang Y., 2010. Le centre d'Art de l'Opéra de Shanghai et l'Opéra national de Paris : étude comparative des stratégies managériales dans le domaine culturel. Thèse de doctorat, Université d'Evry Val d'Essonne, $330 \mathrm{p}$.

Kearney D., 2010. Listening for geography: the relationship between music and geography. Chimera, vol. 25, p. 47-76.

Koch G., 2013. We Have the Song, so We Have the Land: Song and Ceremony as Proof of Ownership in Aboriginal and Torres Strait Islander Land Claims, AIATSIS research discussion paper, $n^{\circ} 33,43 \mathrm{p}$.

Kong L., 2009. Making sustainable creative/cultural space in Shanghai and Singapore. Geographical Review, vol. 99, n 1, p. 1-22.

Larson D., 1980. Music In China Today. The Choral Journal, vol. 21, n 3, p. 13-16.

Lau S.Y.S., Wang J., 2009. Gentrification and Shanghai's new middle-class: Another reflection on the cultural consumption thesis. Cities, vol. 26, $n^{\circ} 2$, p. 57-66.

Lau W.-T., 2005. Twentieth-Century School Music Literature in China: A Departure from Tradition. Journal of Historical Research in Music Education, vol. 27, $\mathrm{n}^{\circ}$ 1, p. 33-48.

Leung B.-W., Xie J., 2011. Students' motivation to study music: The mainland China context. Research Studies in Music Education, vol. 33, n 1, p. 59-72.

Li S., Wang J., 2009. The rhetoric and reality of culture-led urban regeneration. A comparison of Beijing and Shanghai, China. The 4th International Conference of the International Forum on Urbanism, Amsterdam, p. 875-887.

Liang M., 1985. Music of the billion: an introduction to Chinese musical culture. New-York, C. F. Peters Corp, $310 \mathrm{p}$. 
Marton A. M., Wu W., 2006. Spaces of globalisation: Institutional reforms and spatial economic development in the Pudong new area, Shanghai. Habitat International, vol. 30, nº 2, p. 213-229.

Monnet J., 2000. Les dimensions symboliques de la centralité. Cahiers de géographie du Québec, vol. 44, n 123, p. 399-418.

Olivier E., 2004. La petite musique de la ville. Journal des africanistes, vol. 74, $\mathrm{n}^{\circ}$ 1/2, p. 97-123.

Padovani F., 2015. Les transformations sociales du lilong de Tianzifang, à Shanghai. In Franck M., Sanjuan T., Territoires de l'urbain en Asie- Une nouvelle modernité ? Paris, CNRS Éditions, p. 71-84.

Perris A, 1983. Music as Propaganda : Art at the Command of Doctrine in the People's Republic of China. Ethnomusicology, vol. 27, $\mathrm{n}^{\circ}$ 1, p. 1-28.

Picard F., 1998. Espace et musique en Chine. In Chouvel J-M., Solomos M., L'espace : Musique/ Philosophie. Paris, L'Harmattan, p. 311-318.

Pons B., Puel G., Xiaojing J., 2006. Nouvelles mobilités et pratiques sociales : les Starbucks à Pékin. Perspectives chinoises, $\mathrm{n}^{\circ}$ 96, p. 35-45.

Raibaud Y., 2006. Comment la musique vient-elle au territoire ?. Volume!, vol. 5, nº 2, p. 205-209.

Raibaud Y., 2009a. Une géographie socioculturelle. Thèse d'habilitation à diriger des recherches, Université Michel de Montaigne-Bordeaux III, 235 p.

Raibaud Y., 2009b. Musiques et territoires : ce que la géographie peut en dire. Colloque international de Grenoble: Musique, territoire et développement local, $9 \mathrm{p}$.

Reuther N., 2007. Chant et territorialité chez les Secwepemc (Shuswap) : ou comment tracer des frontières par la portée du chant. Cahiers d'ethnomusicologie, vol. 20, p. 147-163.

Richaud L., 2016. Au parc par temps de fête : Fêtes étatiques et performances publiques à Pékin aujourd'hui. L'Espace Politique, n 30, p. 1-23.

Rochot J., 2017. «Un parc à soi » : les parcs, territoires de la vieillesse en Chine urbaine contemporaine. Lien social et Politiques, $\mathrm{n}^{\circ}$ 79, p. 193-214.

Rogers K.-C., 1989. Music Education in the People's Republic of China. Music Educators Journal, vol. 76, n 1, p. 43-45.

Romagnan J.-M., 2003. L'activité musicale in situ au service de l'analyse géographique : l'école de musique du district de moyenne Durance (Alpes-de-Haute-Provence). Méditerranée, vol. 100, $\mathrm{n}^{\circ} 1.2$, p. 23-28.

Roseman M., 1998. Singers of the landscape: song, history, and property rights in the Malaysian rain forest. American Anthropologist, vol. 100, n 1, p. 106-121.

Sanjuan T., 2003. Lieux et nouvelles sociabilités en Chine aujourd'hui. Les grands hôtels à l'avantgarde. In Sanjuan T., Les grands hôtels en Asie: Modernité, dynamiques urbaines et sociabilité. Paris, Publications de la Sorbonne, p. 207-215.

Sanjuan T., 2009. Atlas Shanghai. Paris, Autrement, 88 p.

Sanjuan, T., 2015. Atlas de la Chine. Paris, Autrement, 96 p.

Site web Sina. http://sh.sina.com.cn/news/k/2018-03-29/detail-ifysqfni2069489.shtml

Site web Xinmin. http://newsxmwb.xinmin.cn/wentihui/wtsh/2018/01/28/31355697.html

Seeger A., 2004. Chanter l'identité. Musique et organisation sociale chez les Indiens Suyá du Mato Grosso (Brésil). L'Homme, vol. 171-172, n³ 3. p. 135-150. 
Street J., 1993.Local differences? Popular music and the local state. Popular Music. vol. 12, $\mathrm{n}^{\circ} 1$, p. 43-55.

Trébinjac S., 1990. «Que cent chants rivalisent, qu'une musique éclose » : Étude sur le traditionalisme d'État en Chine. European Journal of Sociology, vol. 31, n 1, p. 60-80.

Wong I., 2007. The music of China. In Nettl B., Capwell C., Excursions in world music. New-Jersey, Prentice Hall, p.7 0-104.

Yue J., 2008. Confucius on Music Education. Nebula, vol. 5, n 1-2, p. 128-133.

Zhonggong Shanghai shiwei bangongting [Bureau général du Comité municipal communiste de Shanghai], 2016. Shanghaishi « shi san wu » shiqi wenhua gaige fazhan guihua [13 ${ }^{\text {ème }}$ plan quinquennal pour la réforme culturelle de de la Municipalité de Shanghai].

\section{NOTES}

1. Les lilong, sortes de courées, correspondent au mode d'organisation des habitations shanghaiennes établi dans les années 1930.

2. Le yueju est un opéra traditionnel originaire de la province du Zhejiang implanté à Shanghai depuis les années 1920. Le huju, cité plus loin, correspond à l'opéra shanghaien à proprement parlé. Le jingju est l'opéra pékinois traditionnel, présent partout en Chine. Le kunqu est considéré comme le plus ancien opéra chinois, et est classé au patrimoine mondial immatériel de l'Unesco depuis 2001.

3. Seule la capacité financière conférée par le statut social est ici prise en compte

4. Le terme de «centralité » fait ici référence à une "qualité attribuée à un espace ». Un espace perçu comme central par une personne, ne l'est donc pas forcément pour une autre. La centralité est à distinguer du « centre ", compris comme "attribut intrinsèque d'un lieu » et qui ne peut donc pas être discuté (Monnet, 2000 : 400).

\section{RÉSUMÉS}

Cet article propose une analyse de Shanghai par ses lieux de chant. Une telle méthode, ancrée dans la géographie musicale, permet de rendre compte de manière originale de la planification et de l'organisation spatio-temporelle et socio-culturelle de la ville. La répartition, l'implantation, l'architecture, la temporalité et la fréquentation des lieux de chant témoignent des politiques urbaines et culturelles et illustrent la structuration et le fonctionnement de la société shanghaienne. L'émergence de pratiques urbaines particulières, la modification de la perception de la ville par ses habitants et le réaménagement de certains espaces publics provoqués par le chant révèlent également de nouvelles dynamiques urbaines et sociales.

The purpose of this paper is to analyse the city of Shanghai through the various places in which people sing. This method of basing research on musical geography is an original way to report both the planning and the spatio-temporal, socio-cultural organization of the city. The distribution, geographic location, architecture, temporality and visits of the singing places evidence the urban and cultural policies, and show how the Shanghainese society is structured 
and operates. The emergence of specific urban practices, the alteration of the way the inhabitants perceive their town along with the reorganisation of some public spaces, which were all triggered by the process of singing, also reveal new urban and social processes.

\section{INDEX}

Mots-clés : aménagement spatio-temporel, appropriation, chant, géographie musicale, Shanghai, organisation socio-culturelle

Keywords : appropriation, musical geography, Shanghai, singing, socio-cultural structure, spatiotemporal urban planning

\section{AUTEUR}

\section{SARAH DEFOIN-MERLIN}

Sarah Defoin-Merlin, sarah.defoin@gmail.com, est étudiante en master 2 Dynamiques des pays émergents et en développement (DynPED) à l'Université Paris 1 Panthéon-Sorbonne. 